Aim(s)/objectives Evaluate:: Documentation of syphilis stage, Treatment, Interval between treatment and first follow-up RPR, RPR 12 months post treatment -determine those with negative RPR; fourfold reduction in RPR or documented as "serofast".

Methods A list of positive serology between April 2013 and March 2014 was generated. Patients managed at clinic were identified and their electronic record reviewed. Descriptive column statistics were used for data analysis.

Results 93 cases of syphilis were managed. Median age 35 years (range 20-74); 82 (88\%) cases male; 47 (51\%) HIV positive. Stage was documented in $45(48 \%)$. Of those with undocumented stage, RPR $>/=1: 8$ in 29,25 were treated with single benzathine-penicillin. 85 received penicillin- based treatment, 48 (52\%) received single dose benzathine penicillin. 8 received doxycycline. Median interval from treatment to subsequent RPR 40 days (range14- 219). 12 months post treatment, 48 (52\%) had negative or "neat" RPR. $58(62 \%)$ a fourfold reduction. In those with a positive RPR, 4 were deemed to be "serofast".

Discussion/conclusion Syphilis continues to be a common infection in men. The majority of patients were treated with penicillin as per BASHH guidelines. There is room for improvement in documenting stage of infection and outcome in those who fail to have a fourfold reduction in RPR at 12 months.

\section{P231 SERVICE IMPROVEMENT REVIEW OF SEXUAL HEALTH SERVICE DNA RATES}

Naomi Kaufman*, Claire Dewsnap, Lauren Theaker. Sheffield Teaching Hospital Foundation Trust, Sheffield, UK

\subsection{6/sextrans-2016-052718.279}

Background/introduction Reductions in sexual health budgets have occurred across England. There is significant focus on the Public Health Outcomes framework and requirements to deliver improved services for less. Sexual health services are examining ways to be more efficient. In 2015 we set out to examine aspects of inefficiency within our service. We highlighted a high 'Did Not Attend' (DNA) rate for Follow up patients as a significant inefficiency. We describe a service review undertaken as part of service improvement plan looking at the reasons and triggers for patients not attending or cancelling their appointments.

Aim(s)/objectives To describe associated factors with not attending appointments when a follow up (FU) appointment has been arranged. To identify solutions to reduce DNA rates to release extra capacity for new appointments.

Methods Focused initial data collection on Doctor follow up stream for November 2015. Aimed to look at whether the following impacted on DNA rate: SMS reminders, Time between booking \& appointment and Reason for follow up

Results The overall DNA rate for Doctor Follow up appointments is $23.7 \%$. Median time from appointment made to appointment either attended or not was the same, 18 days. Patients re-attending for Gonorrhoea and chlamydia treatment, Post exposure Prophylaxis FU, Herpes FU and patients with ongoing symptoms attended well. Patients attending for hepatitis $\mathrm{B}$ vaccination, window period testing, FU for Pelvic inflammatory disease and Chlamydia Test of cure were significantly more likely to DNA. A survey was conducted to ask patients what were the acceptable means of avoiding DNAs.

Discussion/conclusion A total of 165 appointments were missed in November 2015, equivalent to 10 appointments every day. Introducing additional text reminders, using social influence models to increase patient cancellation, were all likely to reduce DNA rate and increase capacity for new appointments.

\section{P232 KEEPING ON TOP OF BV - AN AUDIT OF BACTERIAL VAGINOSIS AND STI SCREENING IN GUM CLINIC ATTENDEES REQUESTING A TERMINATION OF PREGNANCY}

Elspeth Bird*, Christina Micanovic, Catherine McCrann, Aseel Hegazi. St Georges Hospital, London, UK

\subsection{6/sextrans-2016-052718.280}

Background/introduction BASHH guidelines currently recommend universal testing for all GUM clinic attendees and all those undergoing termination of pregnancy (TOP). Screening for and treating bacterial vaginosis (BV) is recommended by BASHH in order to reduce the incidence of post TOP endometritis and PID.

Aim(s)/objectives To audit whether STI screening, to include testing for BV, is being carried out in patients attending a GUM service requesting TOP referral.

Methods Retrospective case note review of 100 consecutive patients seen requesting TOP referral in an Inner London GUM clinic $1 / 1 / 15-1 / 2 / 15$.

Results Case notes of 100 were reviewed. Median age was $25 \mathrm{yrs}$ (range $16-49$ yrs). $21 \%$ of patients $(n=21)$ were of Black ethnicity. 28/50 (56\%) reported previous pregnancy and 19/43 (44\%) previous TOP. Sexual histories were documented in 53\% $(n=53)$ of cases. $43 \%(43 / 100)$ of patients were documented to be asymptomatic. $38 / 100$ patients had an STI screen and of the remainder $37 \%$ were documented to have declined. STI screening included serology testing for HIV/STS in $68 \%$ $(n=26)$ of cases and microscopy for BV/TV in $42 \%(n=16)$. No asymptomatic patients were screened for BV. STI prevalence in those screened was BV 50\% (8/16), Chlamydia 8\% (3/37), Gonorrhoea 5\% (2/37).

Discussion/conclusion Adherence to BASHH guidelines was inconsistent particularly in relation to STI screening and BV screening in asymptomatic women seeking TOP. STI prevalence was relatively high in those screened. A designated clinical proforma may improve documentation and testing rates.

\section{P233 A CASE OF CMV (CANCER MIMICKING VIRULENCE)}

'Johnny Boylan*, ${ }^{1}$ Peter Greenhouse, ${ }^{1,2}$ Patrick Horner. ${ }^{1}$ Bristol Sexual Health Centre, Bristol, UK; ${ }^{2}$ University of Bristol School of Social and Community Medicine, Bristol, UK

\subsection{6/sextrans-2016-052718.281}

Background/introduction Generalised lymphadenopathy and fatigue have a broad differential diagnosis ranging from curable infections to lymphoma. Certain diagnoses can be delayed or missed altogether when patients do not present to sexual health clinics.

Aim(s)/objectives Highlight secondary syphilis as a cause of lymphadenopathy amongst non-sexual health colleagues. Expand syphilis testing beyond sexual health clinics, even in the absence of classical risk factors.

Methods Case report of secondary syphilis which was misdiagnosed despite numerous investigations in primary and secondary care. 
Results A 23 year Caucasian lady was referred to lymphoma clinic by her GP with a three month history of lymphadenopathy and fatigue. She was diagnosed with a primary CMV infection and lost to follow up after her symptoms resolved. One year later she attempted to donate blood. Positive syphilis serology with a low RPR was detected on routine blood screening by the transfusion service. The patient was referred to Bristol Sexual Health Centre where sexual history taking revealed she had a bi sexual partner at the time of her illness who also tested positive for syphilis. An archived blood sample from the time of her illness revealed active syphilis infection with a high RPR.

Discussion/conclusion Secondary syphilis can mimic numerous illnesses. However syphilis testing remains uncommon outside of sexual health clinics for a variety of reasons. A change of culture is required to ensure medical professionals are prepared for routine sexual enquiry and consider syphilis as a potential cause for lymphadenopathy.

\section{P234 EXPLORING SERVICE USER NEEDS AND STREAMLINING DELIVERY THROUGH TRIAGE BY SENIOR CLINICIANS IN AN INTEGRATED SEXUAL HEALTH WALK-IN}

Sophia Davies, Rachel Amherst*, Jane Bush. Northern Devon Healthcare Trust, Exeter, UK

\subsection{6/sextrans-2016-052718.282}

Background/introduction On-going procurement exercises in Sexual Health inevitably require that efficiencies are sought whilst maintaining high quality and equity of access.

Aim(s)/objectives This test of change aimed to inform a streamlining of a city centre walk-in service and to test the feasibility of delivering an integrated service using staff with mixed skill sets. Means were sought to reduce re-attendances to the colocated Contraception and GU Medicine services.

Methods For two weeks in January 2016 patients attending the walk-in service were triaged by a senior dual trained clinician. Triage notes were made and patients were subsequently seen by another clinician who could meet all of their needs. Data was collected regarding staff skillsets, waiting times, patient-perceived versus clinician-assessed needs as well as services actually delivered and staff feedback.

Results 324 patients were seen (236 females, 88 male). Approximately $40 \%$ of women had mixed (GU and contraception) needs, whilst of those believing themselves to have a solely contraception need, half were identified as also having a GU need. A third of patients were symptomatic Two thirds had a sexual health screen. Nursing staff saw $66 \%$ of patients. $80 \%$ of staff reported similar or increased job satisfaction (qualitative data are available).

Discussion/conclusion Service users perceived needs are often less than those assessed by experienced clinicians. Delivering an integrated walk-in service whilst maintaining specialist skills is feasible if triage is effective; missed opportunities are minimised. Work to assess the utility and cost-effectiveness of different types of triage is required

\section{P236 A REVIEW OF A YEAR OF NEW HIV DIAGNOSES AT A SINGLE CENTRE - WHAT CAN WE LEARN? HOW PEOPLE TEST, PRIMARY INFECTION AND HOSPITALISATION}

${ }^{1}$ Sarah Cavilla* ${ }^{1,2}$ Daniel Richardson. 'Brighton \& Sussex University Hospitals NHS Trust, UK; ${ }^{2}$ Brighton \& Sussex Medical School, Brighton, UK

10.1136/sextrans-2016-052718.283
Introduction A large proportion of new HIV diagnoses are incident and hospitalised. HIV testing can be accessed by various means. HIV incidence in UK men who have sex with men (MSM) continues to increase. HIV testing is an important public health intervention to reduce incidence. Locally we have a high prevalence $(8: 1000)$ and an HIV cohort of 2300 mainly MSM.

Methods New diagnoses from January- December 2015 were identified from our local database, clinical records were examined. Data was collected on patient characteristics, medical history, results, the method and location of testing.

Results There were 57 new diagnoses; 50 (88\%) MSM, 4 (7\%) heterosexual females and $3(5 \%)$ heterosexual males. $24(42 \%)$ had tested negative in the previous year, $24(42 \%)$ more than 1 year ago and $9(16 \%)$ had never tested. Testing locations included: 27 (47\%) GUM clinic, 14 (25\%) GP, 5 (9\%) home test kits, 4 (7\%) THT, 3 (5\%) inpatient, 2 (3.5\%) hospital outpatients, $1(1.75 \%)$ sauna and $1(1.75 \%)$ private clinic. Reasons for testing encompassed: 17 (30\%) GUM screening, 10 (18\%) HIV symptoms, 2 (4\%) post exposure prophylaxis, 2 (4\%) partner notification, 2 (4\%) malignancy, 1 (2\%) assault, 1 (2\%) condom break, $1(2 \%)$ intravenous drug use and $1(2 \%)$ insurance. 12/49 (24\%) avidity results were incident. 10/57 (18\%) were hospitalised within 3 months of diagnosis; 3 malignancies, 2 opportunistic infections (Pneumocystis, Mycobacterium avium complex), 1 STI (Shigella), 1 abscess, 1 seroconversion, 1 treatment toxicity and 1 for investigations.

Discussion/conclusion Locally a large proportion of new HIV diagnoses are incident, MSM and hospitalised. Continued innovation is vital in community and hospital admission testing to reduce undiagnosed HIV and incidence.

\section{P237 ASSESSING THE IMPACT OF A HIV TESTING POLICY}

${ }^{1}$ Sarah Allstaff*, ${ }^{2}$ Ewan Barrack, ${ }^{1}$ Ciara Cunningham. ${ }^{1}$ NHS Tayside, Dundee, UK; ${ }^{2}$ University of Dundee, Dundee, UK

\subsection{6/sextrans-2016-052718.284}

Background/introduction Mirroring national data, HIV testing in our local board is failing to reduce the high proportion of late diagnoses. Healthcare Improvement Scotland (HIS) HIV standards 2011 recommended the development and promotion of a written HIV testing policy. A local HIV testing policy was introduced in 2013 based on the UK National testing guideline (BHIVA), recommending routine testing in certain clinical areas, in high risk groups and all individuals with "clinical indicator conditions" regardless of perceived risk. The introduction of the policy was supported by staff training.

Aim(s)/objectives Review the impact of a HIV testing policy on staff knowledge and levels of HIV testing.

Methods In November 2015 a survey was undertaken to question staff awareness of the policy, understanding of HIV testing and levels of HIV testing. The survey was advertised on local websites to healthcare, social work, third sector and substance misuse staff.

Results The survey had 120 respondents, with the largest proportion from Consultant and GP staff. Over $70 \%$ of respondents were aware of the policy. $25 \%$ reported that the policy had changed their clinical practice by increasing their confidence in testing. There has been a corresponding increase in HIV testing figures. Interestingly over $70 \%$ responded that a detailed HIV risk assessment was required even in the presence of a clinical indicator condition. 